\title{
El camino de Colombia a la OCDE ${ }^{1}$
}

\author{
Colombia's road to the OECD
}

\author{
Diana Patricia Arias Henao
}

Resumen: El objetivo de este trabajo es analizar el proceso de adhesión colombiano a la $\mathrm{OCDE}$, con un método deductivo producto de recolección electrónica de fuentes primarias y secundarias. Las características estructurales del diseño metodológico recurren a elementos domésticos y externos del caso colombiano. Los principales hallazgos describen los resultados teóricos y prácticos encontrados entre 2013 y 2018, y dejan abiertas las puertas para la continuación del estudio en cuanto al proceso de ratificación del tratado, en primera instancia, y, en caso de conseguirse de forma positiva, en vías de analizar los impactos y desafíos nacionales, regionales e internacionales de ingresar formalmente al club de los países ricos y de las buenas prácticas. Conclusiones jurídicas, políticas y económicas detallan las implicaciones endógenas y exógenas que anteceden a la configuración del bloque de constitucionalidad.

Palabras clave: Colombia; Economía; Política; Cooperación Internacional; Derechos Humanos

\begin{abstract}
The purpose of this work is to analyze the process of Colombian accession to the OECD, through a deductive method product of the electronic collection of primary and secondary sources. The structural characteristics of the methodological design make use of domestic and external elements of the Colombian case. The main findings describe the theoretical and practical results found between 2013 and 2018, and leave the door open to continue the study in connection with the ratification process of the treaty, in the first place, and, should this be successful, by analyzing the impacts and national, regional and international challenges to formally enter the rich countries' club and good practices. Legal, political and economic conclusions detail the endogenous and exogenous implications, which precede the configuration of the constitutionality block.
\end{abstract}

Key Words: Colombia; Economy; Politics; International Cooperation; Human Rights

\footnotetext{
DOI: https://doi.org/10.24215/23142766e058

${ }^{1}$ Recibido: 03/04/2019. Aprobado: 18/06/2019

2 Postdoctora en Derecho Público por la Universidad de Santiago de Compostela - España y Doctora en Relaciones Internacionales por la Universidad Nacional de La Plata, Argentina. Docente de tiempo completo de la Facultad de Relaciones Internacionales, Estrategia y Seguridad de la Universidad Militar Nueva Granada. Bogotá - Colombia. Diana.arias@unimilitar.edu.co. ORCID: http://orcid.org/0000-0002-4289-5186
} 


\section{Introducción}

Colombia ha decidido seguir y promover los principios y valores esenciales que enarbola la Organización para la Cooperación y el Desarrollo Económico (OCDE), organismo de cooperación internacional con sede principal en París, cuyos idiomas oficiales son el francés y el inglés. El órgano de dirección estratégico y de supervisión es el Consejo, el cual decide por consenso y se compone de un representante de cada país miembro y un representate de la Comisión Europea, y se reúne una vez al año ministerialmente para deliberar sobre asuntos clave y definir líneas de trabajo para la Secretaría. Además, está en permanente interacción con otros organismos como La Organización del Tratado del Atlántico Norte (OTAN).

Originalmente, veinte países de Europa y América del Norte adhirieron a la Convención de París el 14 de diciembre de 1960. La OCDE se constituyó formalmente en 1961 y sucedió a la Organización Europea para la Cooperación Económica (OECE), resultado del Plan Marshall y de la Conferencia de los Dieciséis (Conferencia de Cooperación Económica Europea), que existió entre 1948 y 1960.

Este organismo ha cambiado conforme el contexto internacional y ha debido readaptarse a las nuevas necesidades internacionales. Así como la escena contemporánea transpoló los idearios clásicos, las postrimerías de la Guerra Fría flexibilizaron los estándares de adhesión a la OCDE, convirtiéndola en incluyente e invitando a ingresar a naciones no desarrolladas ni industrializadas a participar en uno de los foros globales más influyentes, cuya sede se remodela para impactar positivamente los procesos de cooperación internacional.

En marzo de 2019, la página oficial de la OCDE informa que son 36 los estados miembros: Australia, Austria, Bélgica, Canadá, Chile, la República Checa, Dinamarca, Estonia, Finlandia, Francia, Alemania, Grecia, Hungría, Islandia, Irlanda, Israel, Italia, Japón, Corea, Lituania, Luxemburgo, Letonia, México, los Países Bajos, Nueva Zelandia, Noruega, Polonia, Portugal, la República Eslovaca, Eslovenia, España, Suecia, Suiza, Turquía, el Reino Unido y los Estados Unidos (OCDE, 2019).

La OCDE aceptó el ingreso de Colombia en mayo de 2018, luego de deliberar 5 años y tras obtener el compromiso colombiano de surtir el proceso constitucional de aprobación y ratificación de la Ley aprobatoria, proceso que encuentra ciertas trabas políticas durante la presidencia de Iván Duque (Portafolio, 2018).

El proceso de adhesión de Colombia a la OCDE terminará cuando la Ley mencionada empiece a surtir efectos jurídicos, esto es, una vez que Colombia deposite el instrumento aprobado y ratificado ante el gobierno francés, quien actúa como depositario de la Convención de la OCDE. Esto convertirá a Colombia en el Estado miembro número 37 del club de los ricos incluyentes, lo que permitirá flexibilizar las leyes mediante el uso de instrumentos de soft law o no obligatorios, como lo son los principios de gobernabilidad corporativa de la $\mathrm{OCDE}$, perfilando así caminos de hard law o derecho vinculante, que se materializan en acuerdos formales tras las negociaciones con la membresía.

Por otra parte, la violencia asociada al conflicto armado ha afectado el crecimiento de la productividad en el pasado y ha impactado profundamente el bienestar y el desempeño económico (Sánchez Galindo y Restrepo, 2009) presentes. El conflicto armado ha destruido muchas infraestructuras, provocando deserción escolar y forzando el desplazamiento de las personas de sus hogares. Ha reducido también la productividad al bajar la calidad institucional, al impactar negativamente en los derechos de propiedad y al dañar la cohesión social 
(Santamaria, Rojas y Hernández, 2013). Todas estas son acciones principales de transformación que deben revertirse una vez Colombia se adhiera formalmente a la OCDE, entre otros.

\section{Planteamiento del problema - ¿Cuáles son los impactos del pro- ceso de adhesión de Colombia a la OCDE?}

\section{Metodología}

Para analizar el proceso de adhesión colombiano a la OCDE, se utilizará el método descriptivo en el análisis de las fuentes primarias y secundarias recolectadas a través de minería electrónica. Se tendrán en cuenta los asuntos endógenos y exógenos del proceso de adhesión para determinar los resultados a nivel teórico y práctico, hallados entre 2013 y 2018, relativos al sector socio-económico, en cuanto al camino de Colombia a la OCDE. EI estudio arroja conclusiones jurídicas, políticas y económicas, las cuales diferencian las etapas previas a la configuración del bloque de constitucionalidad. Esto evidencia los asuntos que Colombia, como próximo Estado miembro de dicha organización, tiene que mejorar. Igualmente se atiende la coyuntura económica que representa la guerra comercial entre Estados Unidos y China, y cómo esto afecta el sector colombiano.

\section{El camino de Colombia hacia el Club de los "Ricos Incluyentes"}

Colombia inició el camino para ingresar a la OCDE de forma oficial en el año 2013. No obstante, desde los inicios del primer mandato de Juan Manuel Santos Calderón en 2010, la adhesión a la OCDE ya resonaba como un reto.

Los principales sectores de acción del organismo se pueden agrupar así:

- Preservación (y constante perfeccionamiento) de la democracia como sistema político,

- Defensa de los derechos humanos y

- Aplicación y custodia de la economía de libre mercado que conlleva liberalizar progresivamente los movimientos de capitales y de servicios, aplicar los principios de no discriminación, trato nacional y trato equivalente, y el respeto por la propiedad privada.

Si bien la arista principal parece girar en torno al aspecto económico, para Colombia no representaría un cambio drástico, ya que desde 1991 el país emprendió constitucionalmente el camino del libre mercado.

Colombia se presenta como una economía competitiva en América Latina y se codea, aún sin carnet, globalmente con los gobiernos del club de los países ricos, de economías democráticas, para buscar respuestas comunes a los retos económicos y sociales, retroalimentándose entretanto a través de cada experiencia y de las buenas prácticas, e integrando políticas locales e internacionales.

Colombia proyecta expandirse económicamente, de la mano de la OCDE, multiplicando las relaciones comerciales multilaterales desde una base no discriminatoria, conforme a las obligaciones y principios de derechos internacional.

La OCDE es un canal importantísimo donde se intercambia información a través de Comités Especializados, grupos de expertos y de trabajo, desde donde brota una fuente de reconocido prestigio de estadística y datos, que establece tendencias y provee previsiones en la mayoría de los sectores de la globalización, en especial, en el económico. 
Asimismo, investiga y produce modelos sociales, comerciales, laborales, ambientales, agrícolas, tecnológicos, fiscales, cooperativos, de gobernanza, transparencia y mercados financieros, entre los principales asuntos que trata. Dicha información, no solo es compartida con sus estados miembros, sino también, con Estados que están en proceso de adhesión, con otros estados no miembros que actúan como observadores regulares de los Comités, y, con estados asociados, que pueden solicitar su membresía permanente previa evaluación positiva.

Incluso, con las adaptaciones del organismo en el tiempo y el contexto, su objetivo se ha tornado feroz en cuanto a la caza de nuevos contactos con países no miembros, al punto de estructurarse para ello la Secretaría de Relaciones Globales (GRS, por sus siglas en inglés), que controla y vigila las orientaciones de tipo estratégico en este campo.

Más de 70 países orbitan las temáticas de la OCDE. Incluso Rusia empezó su proceso de acercamiento desde 2007, pero en 2014, por las consecuencias de la crisis de Crimea, el proceso de adhesión se enfrío, debido a la decisión de suspensión de la OCDE. Igualmente China, India, Indonesia y países africanos, entre otros, interactúan con el organismo de forma constante. Todo en pos de establecer políticas comunes, expandiendo igualmente su relación con la sociedad civil y las empresas privadas.

El presupuesto de la OCDE no permite hacer préstamos, no cuenta con un sistema de cuotas de representantes nacionales, sino que se estructura a través de un sistema de igualdad de oportunidades con personas altamente calificadas. Dispone de unos 330 millones de euros anuales aportados por los estados miembro y las contribuciones nacionales se calculan conforme el PIB nacional. El mayor contribuyente es Estados Unidos, con un $25 \%$ del total presupuestal, seguido de Japón (OCDE, 2019).

\section{Acciones concretas del estado colombiano}

Las opiniones críticas, constructivas y propositivas que se hacen entre sí los miembros de la OCDE promueven la búsqueda de respuestas y caminos comunes que efectivizan la consecución de transformaciones concretas.

El ejercicio de espejo que juegan los estados pone en el banquillo realidades y metas concretas que deben ser atendidas por cada miembro, en sectores tan diversos como amplio es el espectro de la globalización. La presión multilateral voluntaria acarrea, sin duda alguna, una mejor autocomprensión del estado de la cuestión nacional, los efectos y las oportunidades que representa el contexto internacional y la posibilidad de caminar conjuntamente no solo en la aplicación de soluciones sino en el diagnóstico de las realidades.

El proceso de ingreso a la OCDE ha activado, conforme lo expresó en medios Juan Manuel Santos Calderón, reformas institucionales y ha desencadenado reflexiones internas muy importantes: la OCDE, afirma el ex presidente, hace bien en voltear sus ojos hacia nuestra región. Es una región que podrá beneficiarse enormemente de generalizar los conceptos que promueve la OCDE de gobernanza, transparencia e inclusión: Latinoamérica necesita a la OCDE y la OCDE necesita a Latinoamérica.

La heterogeneidad en el nivel micro en materia de productividad es común en el conjunto de los países de la OCDE (OECD, 2015b); en Colombia, la dispersión es particularmente alta (Brown et al., 2013). Aunque las empresas colombianas estén lejos de operar en los 
niveles de productividad de las empresas más avanzadas a nivel mundial, algunas empresas son altamente productivas hasta a escala mundial (Global frontier; Brown et al., 2013).

La informalidad tiene implicaciones importantes para la productividad, el crecimiento económico y la desigualdad de los ingresos (OCDE, 2009; Loayza et al., 2009 y Dougherty y Escobar, 2013). Deficiencias en la infraestructura material reducen la productividad de los factores y el rendimiento de la cosecha, debilitan la competitividad de mercado y limitan la integración espacial y temporal (Fan, 2004; Pinstrup-Andersen y Shimokawa, 2006). Adicionalmente, la imposibilidad de individuos con un entorno socioeconómico pobre para acceder a una educación de calidad ( $y$, por tanto, desarrollar su capital humano) provoca una perpetuación de la desigualdad de ingresos, lo que reduce el crecimiento económico (Causa y Johansson, 2010).

Colombia adecuó la regulación de los procesos de adquisición de créditos para programas y proyectos dirigidos a la exportación de la producción nacional, bajo la premisa de una reducción notable de la corrupción. Asimismo se ajustó la normatividad del sector agrícola (infraestructura para una mejor productividad y administración de lo público) y se previó la actualización jurídica y práctica en beneficio del movimiento de capital foráneo en el país, con el objeto de dinamizar el flujo de las inversiones y de crear más y mejores empleos. Se creó la Red Nacional de Consumo Seguro, la que beneficia directamente a los consumidores nacionales frente a posibles abusos del mercado de la mano de la Superintendencia de Industria y Comercio (SIC). Por último, se avanzó significativamente en materia de estadística de la administración pública, con la modificación de las estructuras de las juntas directivas de las empresas del Estado, mediante la creación de una nueva política de propiedad estatal y la consecuente actualización del Código País sobre los estándares de gobierno corporativo (OCDE, 2018).

\section{Observaciones económicas de la OCDE sobre Colombia}

Desde el año 2016 se ha desacelerado el consumo de bienes y la inversión pública responden a políticas monetarias y fiscales más estrictas. Como consecuencia de ello, el desempleo subió levemente (Banco Central, 2016). La inclusión financiera ha sido una prioridad del gobierno, mediante el otorgamiento de microcréditos a los más pobres, la difusión del uso del sistema bancario formal, el aumento del uso de medios de pago electrónicos, y facilitando el acceso a los servicios financieros (Karpowicz, 2014).

Por su parte, la OCDE referencia como positivo el diseño e implementación de las políticas macroeconómicas del Estado colombiano, al considerar que ellas brindaron la plataforma para caminar por el sendero del crecimiento sostenido a pesar del declive total de los commodities.

En su informe semestral de perspectivas, este organismo anunció para Colombia un incremento del PIB de hasta el 3,3 \% en 2019, debido a la multiplicación del sector de las inversiones, así como la evolución gradual de las infraestructuras de diversos sectores económicos y comerciales. Además se informa acerca de los beneficios que han traído a las empresas la reducción impositiva y el alivio del repunte de los precios del petróleo. La OCDE considera adecuada la política monetaria, así como la reducción de la inflación al 3 \%, logrando las finalidades de la política acomodaticia. En cuanto a la política fiscal, la OCDE destaca que esta obedece a un planteamiento prudente que permite la proyección de reducir 
el déficit del gobierno al $1 \%$ del PIB en 2022. Sin embargo, la OCDE insta a Colombia a mejorar los asuntos relacionados con la eficiencia del gasto y aumentar los ingresos para cumplir con la regla fiscal. Se requiere equilibrar las fuertes asimetrías que se presentan a nivel regional, mejorando las vías de acceso y puertos, lo que redundaría en beneficio de la creación de nuevas plazas laborales y aumentaría el nivel de competitividad empresarial. Se debe prestar especial atención a la situación migratoria venezolana en territorio colombiano, cuyo flujo es elevadísimo y constante, lo que puede aumentar el gasto. No obstante, en un buen cálculo, se podría potenciar, en un mediano plazo, el crecimiento económico (Antolinez, 2018). Migración Colombia informó que marzo de 2019 cerró con la cifra de 1.260.594 venezolanos en Colombia, con un ingreso de 85.851 connacionales en el último trimestre (Morales, 2019).

En cuanto al sector tecnológico, se espera que el nuevo programa 4G complete $5.892 \mathrm{~km}$ de carreteras vía APPs, lo que requiere una inversión de USD 10.700 millones (Bell y Schipani, 2015). La OCDE estudia el camino de beneficios que brinda la revolución tecnológica informática al crecimiento económico y ayuda a los gobiernos a crear políticas económicas y armonizar las transacciones internacionales.

Si no se saca a los colombianos de la informalidad, la tendencia será la inequidad y el ensanchamiento de la brecha de la disparidad de ingresos (Cournede et al, 2016, Adalet Mc Gowan et al, 2017). Asimismo, una mayor inversión en la educación de la primera infancia puede reducir las tasas de deserción en la secundaria, mejorar el rendimiento estudiantil y achicar la brecha en el resultado del aprendizaje (Heckman, 2006; OECD, 2011). Otro aspecto que resalta el organismo tiene que ver con trabajos realizados en tiempo no remunerado, como tareas de cuidado y asistencia y labores domésticas (Miranda, 2011; Ferrant, et al 2014). Urge reducir el $21 \%$ de madres cuyos hijos no se encuentran bajo el cuidado formal (Frey et al, 2017). Nuevas oportunidades de educación y formación fortalecen las competencias de la mano de obra femenina (Bergemann y Van den Berg, 2008).

La educación avanzó hacia la jornada única, con incentivos para maestros, un mejoramiento de la infraestructura educativa, la cobertura y gratuidad en la educación básica, media e incluso terciaria, a partir de programas como "Ser Pilo Paga". Se creó una política nacional de residuos sólidos y en relación con el riesgo de productos químicos, negocios verdes, y la reducción del uso del plástico y otros materiales de difícil descomposición. Igualmente, se han fortalecido las políticas de transparencia (Decreto 270 de 2017), dando participación directa a la población civil en los procesos para la obtención de licencias y permisos del sector productivo nacional. Las condiciones del país son favorables, ya no solo para la economía extractiva, sino también para el desarrollo de actividades agroindustriales y de carácter netamente industrial, tecnológico y del sector servicios. Es momento de abandonar la tradicional mirada de corto plazo para adentrarse en proyectos innovadores que faciliten la diversificación, tanto en la producción como en los mercados destino, a los que esa mirada se dirige (OCDE, 2018).

La OCDE igualmente resalta que la creación de un sistema coherente y completo de integridad pública en Colombia es esencial para salvaguardar la participación inclusiva en el avance socioeconómico. Resulta crucial promover y edificar la legitimidad del Estado, mediante la concreción de los fines establecidos en el Acuerdo General para la Terminación del Conflicto y la Construcción de una Paz Estable y Duradera.

Para lograrlo es necesario integrar el desarrollo a las políticas del postconflicto, coordinando las diferentes herramientas institucionales del sistema colombiano de integridad 
pública: la Comisión Nacional de Moralización (CNM), que controla la política nacional de integridad y reúne a sus actores y que debería incorporar al Consejo Nacional Electoral (CNE) y al Departamento Administrativo de la Función Pública (DAFP).

El progreso en la agricultura ha estado estrechamente asociado a la infraestructura rural (Lozano-Espitia y Ramírez-Villegas, 2016). La estructura de mercado de la industria del transporte puede ser una determinante clave en los costos de flete (Hummels, Lugovskyy, y Skiba, 2009).

\section{Tabla No. 1: Desafíos para el Estado colombiano sujetos a respuestas multilaterales}

- El crecimiento de la productividad es bajo y la brecha entre ricos y pobres es de las más altas de América Latina

- $\quad$ Alto grado de informalidad

- Brecha de género

- Movilidad social escasa

- Años de conflicto armado

- Regulaciones locales estrictas

- Distorsiones en el sistema impositivo han creado disparidades en la productividad y en el acceso a servicios básicos en todas las regiones

\section{Respuestas comunes de la organización a desafíos similares}

- Simplificar los procedimientos para el registro de empresas y la afiliación de los trabajadores a la seguridad social

- Mejorar programas para el mercado laboral

- Extender la educación de la primera infancia

- Aumentar la calidad de la educación

- Impulsar la inclusión, la movilidad social y los estándares de vida.

- Cobertura mayor y más accesible para niños, adultos mayores y personas con discapacidad

- Aumentar la redistribucion de ingresos a través del sistema de impuestos y transferencias.

- Mejorar la infraestructura

- Reducir costos para el comercio

- Mejores incentivos para que las empresas inviertan en I+D

- Establecer vínculos más fuertes entre el sector privado y las instituciones de investigación favorecería la innovación

- En algunos sectores como el ferroviario, la electricidad y el vial, son necesarias mayor competencia y menor regulación para apoyar la inversión privada

Fuente de elaboración propia.

Ahora bien, pese a los esfuerzos y avances que logró el gobierno de Juan Manuel Santos Calderón para llevar a cabo acciones concretas que permitan el ingreso de Colombia a la OCDE, el proceso se está enturbiando con las acciones del Ejecutivo que lidera el presidente Iván Duque, quien desde el año pasado ha sido renuente en agilizar el proceso de aprobación de la ley respectiva.

Igualmente, el líder del Estado colombiano ha brillado por su ausencia en la interacción con el organismo. Solamente se ha reunido una vez con la Secretaría General de la OCDE. Si bien se deben firmar los convenios de adhesión y de privilegios e inmunidades, 
sostiene el gobierno que aún no está definida de forma detallada y expresa la participación colombiana. Un status quo que debe alterarse con la actividad del Congreso mediante la aprobación del acuerdo de inmunidades y privilegios que establece las reglas de los funcionarios de la OCDE, con respecto a temas jurídicos, acciones judiciales, operaciones cambiarias, impuestos y aranceles, así como debe contar con el visto bueno constitucional de la Corte. Se estima que todo se sustancie para agosto de 2019 (Portafolio, 2019).

Más que los asuntos económicos son los asuntos prácticos de defensa de los derechos humanos los que se tornan más espinosos para la Colombia de 2019, frente a la OCDE, pues denota en un sistema con fallas estructurales en su sistema democrático.

\section{Situación de derechos humanos en Colombia 2018}

Para la Colombia examinada entre 2017 y 2018 en materia de derechos humanos, es preciso destacar la observación que hace Amnistía Internacional sobre la población civil, especialmente los pueblos indígenas, las comunidades afrodescendientes y campesinas, y los defensores y defensoras de los derechos humanos, pues se indica que estos grupos siguen constituyendo los sectores de la población más afectados por el conflicto armado. Aunque las cifras oficiales indicaban que, desde el inicio de las negociaciones y hasta la firma del acuerdo de paz en 2016, había disminuido la cantidad de civiles muertos en acciones militares en las que estaban involucradas las Fuerzas Armadas Revolucionarias de Colombia (FARC) y las fuerzas de seguridad colombianas, el conflicto armado persistía en 2017 y en algunas partes del país parecía haberse intensificado. Continuaba la preocupación ante la impunidad por los crímenes cometidos durante el conflicto armado. Los cuerpos de seguridad hacían un uso excesivo de la fuerza, que a veces causaba muertes de civiles. Persistía la violencia contra las mujeres, en especial, la violencia sexual (Amnistía Internacional, 2017).

Asimismo, se reveló que la Unidad para la Atención y Reparación Integral a las Víctimas, creada en 2011 mediante la Ley 1148, registró un total de 8.532.636 de víctimas durante los cinco decenios que duró el conflicto armado con las FARC. Entre ellas se incluían 363.374 víctimas de amenazas, 22.915 víctimas de delitos sexuales, 167.809 víctimas de desapariciones forzadas, 7.265.072 víctimas de desplazamientos forzados y 11.140 víctimas de minas terrestres antipersonal. Los delitos contra 31.047 víctimas del conflicto armado se registraron por primera vez entre enero y octubre de 2017 (Amnistía Internacional, 2017).

El 2018 terminó con un acuerdo de paz en curso con las FARC, pero con la intensificación del conflicto armado en las zonas dejadas por los farcianos y que son ámbito de enfrentamiento entre los guerrilleros del Ejército de Liberación Nacional (ELN), los paramilitares mutados en Bandas Criminales, y las disidencias de las FARC, las Fuerzas Militares y la Policía colombianas. Cabe destacar que, en Colombia, las guerrillas son "percibidas como peligrosas y que tienen un elemento en común de segregación socio espacial” (CPAT \& Parces, 2017, p. 9).

El Sistema de Verdad, Justicia, Reparación y No Repetición, que creó la Jurisdicción Especial para la Paz (JEP), diseñó los mecanismos jurídicos para investigar a los actores del conflicto armado específico, incluidos los militares. Casi dos mil integrantes del Ejército se han sometido a la JEP. No obstante, por las objeciones realizadas por el presidente Iván Duque al marco normativo de la JEP en marzo de 2019, así como por otras cuestiones políticas, se ha perdido estabilidad jurídica respecto a este proceso alternativo de Justicia. 
Ahora bien, los innumerables conflictos armados territoriales sucedidos en Colombia fuerza a repensarlos desde una reingeniería de políticas publicas, como herramientas para la no repetición sistemática de la violencia:

"La exigencia es bastante alta, sobre todo en una sociedad en la que la resolución de conflictos a través del uso de la violencia, generalmente armada, se ha naturalizado; de manera que hay cierta ceguera que impide vislumbrar las conexiones, las intersecciones y las causas que producen los conflictos. Al mismo tiempo, esta condición de normalización ha ensombrecido la proliferación de otros conflictos que no parecen anexos al desarrollo del conflicto sino una consecuencia directa de la misma. Esos otros conflictos se han enquistado en las lógicas mismas de la sociedad, de manera que el tratamiento ofrecido por la justicia ha sido el de reprender sin reflexionar sobre las múltiples conexiones que se han configurado (...) Los efectos de la negociación en la Habana han dejado entrever que la construcción de paz es una tarea que exige repensar los marcos teóricos con los que se estudia a la justicia, la forma en la que se gobiernan las regiones y el modo en que se organizan las comunidades en los territorios, más allá de las condiciones mismas, en las que se vivía en medio de la confrontación armada. Asimismo, se ha puesto en cuestión la naturaleza de la continuidad del conflicto y se ha abierto una pregunta por la forma en la que una organización armada al margen de la ley puede negociar su desarme con el Estado y su reincorporación a la sociedad civil" (Arias \& Idrobo, 2018, 2-3).

Por su parte, el Informe Anual de marzo de 2019 del Alto Comisionado de las Naciones Unidas para los Derechos Humanos en Colombia subraya la importancia de garantizar los derechos humanos como vía para lograr una paz sostenible y duradera. Destaca los desafíos específicos que plantean la defensa de los derechos humanos, la lucha contra la impunidad y la corrupción, el incremento de la violencia y la necesidad de reducir la brecha de desigualdad en el disfrute de los derechos económicos, sociales y culturales, particularmente en zonas rurales. Igualmente señala la responsabilidad histórica del gobierno de Iván Duque, para continuar con la implementación del Acuerdo y para avanzar en la construcción de un país en paz y equitativo, donde toda la población tenga garantizado el pleno acceso a los derechos humanos (ONU, 2019).

La ONU en su informe señala que los principales responsables de los homicidios dolosos en Colombia son los miembros de estructuras paramilitares desmovilizadas (40 \%), individuos no afiliados a ningún grupo criminal o grupo armado ilegal (18\%), integrantes del ELN (8\%), integrantes del Ejército Popular de Liberación (EPL) (4\%), miembros de la fuerza pública (5\%), antiguos miembros de las FARC-EP que no se acogieron al proceso de paz ( 8 $\%)$ y personas por determinar (17\%). Asimismo, el Informe del Alto Comisionado revela como preocupante las agresiones a los miembros de la comunidad LGBTI. La tasa de impunidad en el juzgamiento de los homicidios dolosos es una de las más altas en la historia de Colombia, la que oscila entre el $86,58 \%$ y el $94,30 \%$ de los casos que son reportados a las autoridades respectivas. Igualmente el informe coincide con las necesidades de alcanzar los compromisos adquiridos por Colombia en la Agenda 2030 para el Desarrollo Sostenible, pues Colombia sigue siendo el país más desigual de la región. Otra razón de inequidad es en materia de género, pues en Colombia las mujeres representan más de la mitad de la población (50,6 \%); sin embargo, su participación laboral es un $20 \%$ menor que la de los hombres, 
a pesar de contar con niveles más altos de educación superior, y la brecha salarial es del $18,7 \%$ (ONU, 2019).

Un asunto clave para sacar de la informalidad e ilegalidad a la población y equiparar gradualmente la brecha de desarrollo será abordar el asunto de drogas ilícitas, acorde a los avances internacionales sobre las políticas antidrogas, que no se satisfacen con respuestas unidimensionales de seguridad y que propenden por soluciones multidimensionales que atiendan además asuntos relacionados al desarrollo, la salud pública y la observancia del derecho internacional de los derechos humanos. La sustitución voluntaria de los cultivos ilícitos, priorizada con un programa de subsidios, asistencia técnica, proyectos productivos y el impulso de la reforma rural integral, favorece la sostenibilidad de los esfuerzos de sustitución, así como, el goce efectivo de los derechos humanos de la población que habita en las zonas de producción de cultivos ilícitos. No obstante, los esfuerzos realizados por la Dirección para la Sustitución de Cultivos llícitos se ven mermados por la inefectividad en los pagos de los subsidios y la implementación de las fases posteriores del proceso de sustitución (seguridad alimentaria, asistencia técnica y proyectos productivos) (ONU, 2019).

La participación política de las FARC se cristalizó cuando el Acuerdo otorgó 10 curules dentro del Congreso de la República. Es hora de que la presencia del Estado no siga limitándose a la presencia de la fuerza pública y de ahondar en el disfrute de los derehos humanos de la población, incluidos el acceso a la justicia y a la seguridad, lo que estimulará el desarrollo económico. Esto rompería con los ciclos crónicos de pobreza multidimensional que es aprovechada por grupos armados ilegales y de criminalidad común. La Contraloría General de la República estimó los costos de la corrupción colombiana en 2017 en el 21 \% del presupuesto nacional del año 2018 (aproximadamente 16.000 millones de dólares). La policía nacional informó que en 2018 el 49,5 \% de los municipios aumentó su tasa de homicidios. Por su parte, el Alto Comisionado detalló que en 2018 las masacres aumentaron un 164 \%, las cuales pasaron de 11 casos en 2017 a 29 en 2018, así como también se produjo el aumento de asesinatos de líderes sociales. Todo esto llama al cambio de diseño de la política de seguridad, por una que cuente con un enfoque de derechos humanos y asegure la plena participación de comunidades así como la coordinación eficaz de las instituciones del Estado (ONU, 2019).

Foucault puede ser clave para este análisis de poder. En sus textos, este autor detalla que el poder existe dentro de una matriz compleja de relaciones que exceden a las figuras estatales y que lo ubican en un centro descentralizado. El ejercicio del poder no es simplemente una relación entre parejas, individuales o colectivas; se trata de un modo de acción de algunos sobre algunos otros. Sólo existe el poder que ejercen unos sobre otros. La relación de poder puede ser el efecto de un consentimiento permanente o anterior, pero no es por naturaleza la manifestación de un consenso (Foucault, 1998)

\section{Hallazgos}

Al terminar la Guerra Fría, Estados Unidos desde sus considerandos de política hemisférica frente a Latinoamérica, no varió de forma significativa:

"la visión sostenida años atrás desde Washington, lo que varió fue el enemigo a enfrentar, al extinguirse toda la atención internacional que se centró en el comunismo y sus focos alrededor del mundo. En los noventa, 
el detrimento se hizo latente, en la dimensión social. Sin embargo, junto con el proceso globalizador, la agenda internacional de tipo vertical comenzará a horizontalizarse, mediante el reconocimiento de nuevas variables, sin descartar a la seguridad, mediante un tratamiento menos monocausal (...) Además de institucionalizar la imposibilidad de separación de las políticas domésticas y las exteriores, dando como resultado que la estabilidad de los países sea una consecuencia de la influencia del país potencia. En síntesis, se trata de una época de instauración neoliberal, que pretendió, infructuosamente hasta ahora, el establecimiento de una economía de mercado" (Arias, 2008, p.16).

Según la OCDE la economía mundial en 2019 solo crecerá en un 3,3\% a causa de tensiones comerciales y por la alta incertidumbre política. La totalidad de las economías del G20 tiene un crecimiento proyectado a la baja, en especial, los Estados ubicados en la eurozona (-0,8 puntos a $1 \%)$ y el Reino Unido (-0,6 puntos a $0,8 \%)$. En caso de sucederse la separación del Reino Unido, perderá un $2 \%$ del PIB por dos anualidades, por el efecto de la aplicación de los derechos arancelarios de la Organización Mundial del Comercio (OMC). Las barreras comerciales han ralentizado fuertemente el comercio mundial, en especial, ha disminuido notablemente el crecimiento chino, el que hace tiempo ya dejó de protagonizar las dos cifras. El organismo prevé un crecimiento del 6,2\% este año (frente al 6,3\% en noviembre). Una caída de dos puntos del crecimiento del PIB en China supondría un recorte del crecimiento mundial de 0,4 puntos porcentuales, según la organización (CM\&, 2019).

Sin duda, el establecimiento definitivo del modelo de desarrollo neoliberal es incuestionable y difícilmente modificable. Dicha castración soberana para decidir con posibilidades un esquema de desarrollo propio implica alabar un modelo primario exportador que, desde los noventa, enfatiza la extracción de recursos naturales de poco valor agregado y, por ende, baja la generación de empleo e ingresos, con lo cual se haría más difícil eliminar la profunda iniquidad existente. Es por ello que, para un sector doctrinal, los costos de ser parte de la OCDE están muy por encima de los beneficios, por lo que la negación de su acceso no implicaría, en términos netos, un perjuicio para el país (Zerda, 2018).

\begin{tabular}{|l|l|}
\hline Avances del camino de ingreso a la OCDE & Falencias o áreas de mejora \\
\hline $\begin{array}{l}\text { Se establecieron marcos jurídicos que representan } \\
\text { seguridad jurídica para los inversores extranjeros y } \\
\text { las políticas públicas producto de las reformas ins- } \\
\text { titucionales emprendidas por el Estado en diver- } \\
\text { sos sectores. }\end{array}$ & $\begin{array}{l}\text { Fortalecer el emprendimiento, la cultura de la } \\
\text { legalidad y crear los mecanismos suficientes } \\
\text { para garantizar la efectiva igualdad de oportuni- } \\
\text { dades. Aimismo, diseñar una estrategia de di- } \\
\text { versificación de aliados comerciales no tradicio- } \\
\text { nales. }\end{array}$ \\
\hline $\begin{array}{l}\text { Crecimiento de productividad, disminución de las } \\
\text { brechas sociales y fortalecimiento de la competen- } \\
\text { cia comercial internacional. Muestra de garantía } \\
\text { de que la gestión gubernamental con nuevas nor- } \\
\text { mas y procedimientos de carácter multilateral son } \\
\text { y serán posibles. }\end{array}$ & $\begin{array}{l}\text { Mejorar sus resultados comparándose con paí- } \\
\text { ses céntricos, conforme a las mejores prácticas } \\
\text { internacionales, a partir del deseo de salir de la } \\
\text { periferia, con acciones concretas para alcanzar } \\
\text { y superar los cometidos iniciales de la mano de } \\
\text { los Estados miembro del organismo internacio- } \\
\text { nal. }\end{array}$ \\
\hline
\end{tabular}


Modernización de prácticas empresariales, fortalecimiento de las superintendencias de Sociedades, Financiera, y de Industria y Comercio; expedición de la ley de conglomerados financieros; intercambio de información tributaria y normas de gobierno corporativo. Exclusión de ministros de las juntas directivas de empresas estatales y se ha promovido la competencia.
Considerar otro tipo de políticas comerciales como las del consenso de Beijing o al menos no esclavizarse con las del consenso de Washington, atendiendo los cambios estructurales de la nueva era económica. profundizar su inserción en la economía internacional.

Profundizar la tecnificación de la autoridad estadística nacional.

Tabla No.2. Fuente de elaboración propia.

Si bien la calidad de vida de los colombianos mejoró desde el año 2000 y se encuentra por encima del promedio de la OCDE, para enero de 2019 la tasa de desempleo fue del $12,8 \%$, lo que constituye un aumento de un punto porcentual con respecto al año 2018 $(11,8 \%)$ (DANE, 2019).

Para la OCDE, la economía de Colombia depende cada vez más de la extracción de recursos naturales. En 2017, la producción primaria y la minería representaron un $80 \%$ de las exportaciones, un $10 \%$ más que en 1991. El sector manufacturero está perdiendo importancia y competitividad. La productividad no aumenta, la brecha laboral es inmensa y solo se desarrollan algunos territorios. La inversión en investigación y desarrollo (I+D) de Colombia se sitúa en un $0,25 \%$ del PIB, cifra 15 veces inferior al promedio de la OCDE y muy por debajo de la del país que más invierte en I+D de esta región, Brasil (1,2 \%) (Nuevo Siglo, 2019).

La Política de Desarrollo Productivo para 2016-2025 crea un proceso consultivo con todas las regiones, para equiparar los desarrollos territoriales conforme sus necesidades y recursos, pero dan un salto hacia adelante al determinar evitar la reunionitis. Esto es fundamental, pues se requiere concentrar los esfuerzos en cambios concretos y no disiparse en los anaqueles de la dispersión planificativa. Para lograrlo, es vital la reestructuración en la planificación, función central. Es necesario dejar de lado la cultura del pupitre y enfocarse en la consecución de los resultados, potenciando los incentivos a la transformación concreta y gradual y no a la eterna redacción de documentos que se amontonan unos sobre otros. Al equiparar los desarrollos territorial, las posibilidades de las regiones producirán un aumento de la productividad y la diversificación constante. Es esencial entonces recalcar la urgencia de desburocratizar los trámites y agilizarlos mediante la mejora de la infraestructura comunicacional. Finalmente, es preciso el fortalecimiento de la política comercial colombiana bajo la correcta implementación de transferencia tecnológica y cooperación técnica (Nuevo Siglo, 2019).

La OCDE informó a través de su Política de Transformación Productiva que en Colombia, entre 2000 y 2017, se duplicó el PIB per cápita y la economía creció a un ritmo anual promedio del 4,3\%, el doble de la tasa de crecimiento de América Latina. El índice de pobreza descendió del 50 \% al $28 \%$. En 2017, aumentó la inversión extranjera directa un $59 \%$ del PIB, de las más altas en Latinoamérica y por encima del promedio de la OCDE, en el 48 \% (OCDE, 2019).

Un hallazgo irrefutable es el potencial que ofrece el sector del turismo en la llamada época del posconflicto, donde Colombia empieza a destacarse en el sistema internacional, ya no sólo por sus historias de drogas y violencia, sino de por la belleza infinita de su gente y su biodiversidad. No obstante, más allá de la inseguridad, nos azotan dramas tan contundentes como una elevada cultura de la contaminación. Escenarios que deben adaptarse para innovar en nuestras inmensas posibilidades de desarrollo verde. 
Existe una necesidad imperiosa de la economía colombiana de transformarse sin tantos miramientos su escenario productivo, para aprovechar sus posibilidades no tradicionales, escalando socialmente, en el modelo económico.

La OCDE ha solicitado a Colombia finalizar el proceso de adhesión, en lo posible, el próximo 23 de mayo de 2019, y le dio un espaldarazo al Plan Nacional de Desarrollo (PND) radicado por el Gobierno Nacional. Asimismo elogió los esfuerzos del gobierno Duque para alinear el plan con los objetivos de desarrollo sostenible (Portafolio, 2019, b).

Colombia, como economía emergente, realizó 23 reformas tangibles durante el proceso de adhesión a la OCDE, la que espera su pronta ratificación (Botero, 2019). La coyuntura económica internacional se tambalea entre el posible tránsito de países alineados con el consenso de Washington al de Beijing. El declive económico de las principales democracias occidentales que siguen desde hace 3 décadas las políticas económicas del Consenso de Washington, bajo la dirección del Banco Mundial y el Fondo Monetario Internacional, hace estrechar los lazos entre China y América Latina (Villar, 2017).

Los mil trescientos millones de chinos representan $16,5 \%$ de la economía mundial (frente al 16,3\% de Estados Unidos). El Banco del Pueblo chino cuenta con 4 billones de dólares en reservas internacionales, y el yuan es la séptima divisa más usada para pagos globales. Sin embargo, China sólo tiene el 3,81\% de los votos en el FMI (Estados Unidos participa con el $16,74 \%$ ), lejos de su peso real en el contexto económico mundial, por lo que China creó el Banco Asiático de Inversión en Infraestructura (BAlI), que cuenta con miembros como BRICS como Alemania, Reino Unido, Francia, Italia, Países Bajos, Australia, España, Corea del Sur e Israel, entre los más llamativos. China materializa la teorización del Consenso de Beijing y su nueva diplomacia financiera, menos invasiva que la estadounidense, y con prácticas de crecimiento enfocadas en inversiones extranjeras directas en los sectores de infraestructura, manufactura, tecnología, energía y telecomunicaciones; y obtiene un promedio de 200 mil millones de dólares anuales, pisándole los talones a Estados Unidos con 367.000 mil millones y horizontalizando su hegemonía unipolar. China, Rusia y BRICS representan aliados reales y estratégicos para América Latina, sin que se repita la condena de los aliados-posesivos (Serrano, 2015).

China informa en sus medios que ha iniciado la guerra del pueblo contra la avaricia y la arrogancia de los Estados Unidos luego de la imposición arancelaria liderada por Trump a los productos chinos que se aproxima a los US\$200.000 millones. El presidente Xi invoca a Mao Zedong, mientras sus críticos denuncian concentración de poder y culto a su propia personalidad, así como se alegan sistemas de vigilancia masiva de la población, desaceleración del crecimiento, aumento de la deuda, falta de inversión privada, escasez de créditos, pérdidas de empleos, jornadas laborales desmedidas, detenciones, encarcelamiento de la minoría musulmana china en campos de detención en Xinjiang y asuntos urgentes de abastecimientos en granos y carne de cerdo. Todo ello se suma a las presiones estadounidenses para no permitir el fomento a la inversión en alta tecnología china, principalmente la 5G. EI 2019 es un año trascendental para China: se conmemoran 70 años desde que Mao fundó la República Popular China, 60 años del levantamiento fallido contra el gobierno chino en el Tíbet y la primera década de los disturbios étnicos en los que murieron cientos de personas en Xinjiang, así como los 30 años de la masacre de Tiananmen (BBC News, 2019). Definitivamente es el año del cerdo, en el que se están dejando vislumbrar cambios trascendentales.

La mal Ilamada guerra comercial entre Estados Unidos y China representa una verdadera crisis no solo a nivel económico bilateral sino también sistémico y se extiende a otros sectores 
que atienden a las relaciones internacionales así como las propias particularidades domésticas. Las decisiones políticas trumpianas, prorrogadas o no, han derivado severas condiciones en el sector de la tecnología especialmente, como lo ejemplifica el caso de la nueva colaboración de Google con Huawei luego del retraso por 90 días de la aplicación de sanciones impuestas por el Departamento de Comercio estadounidense al gigante chino por haberlo incluido en su lista negra, lo que imposibilita a las compañías estadounidenses adquirir componentes tecnológicos sin permiso del Gobierno.

La decisión de sancionar ha sido fundamentada a partir del riesgo a la seguridad nacional estadounidense mediante la invocación de la Ley de Poderes Económicos de Emergencia Internacional, que da al presidente la autoridad de regular el comercio en respuesta a una emergencia nacional que amenace al país. En este caso, la amenaza nacional es el uso de los dispositivos Huawei. Por la adhesión a la lista negra, Google suspendió la transferencia de productos de hardware y software, excepto aquellos cubiertos por licencias de código abierto, a Huawei. Asimismo, Intel, Qualcomm, Xilinx y Broadcom también pusieron en marcha planes para restringir sus suministros. Todo ello hasta que unos días después se concediera una prórroga de 3 meses antes de imponerse las restricciones comerciales. Por los próximos 90 días podrá Huawei realizar actividades operacionales para las redes existentes, el soporte de servicios móviles existentes y la investigación en ciberseguridad para el mantenimiento de la integridad y fiabilidad de las redes y equipos existentes. Huawei afirma que la medida limita al consumidor estadounidense a productos caros y de menor calidad, rechaza el avance a redes $5 \mathrm{G}$ y perjudica a sus propias empresas. Además, anuncia graves consecuencias legales (El Mundo, 2019). Una de las razones que más incomoda la seguridad nacional de los EEUU es precisamente que sus organismos de seguridad no pueden acceder a la información de los usuarios de los dispositivos Huawei, pero los organismos de inteligencia chinos sí (Lioman, 2019).

La guerra comercial entre China y Estados Unidos ya tiene dos perdedores: sus protagonistas. La OCDE y el FMI han advertido que este conflicto abierto puede provocar la recesión global. Han informado además que al incrementar bilateralmente los aranceles hasta el $25 \%$, las exportaciones chinas caerían un $25 \%$ y las de Estados Unidos hacia Pekín, un 36\%. China y la Unión Europea aguantarían mejor, porque captarían parte de ese desvío comercial, pero los países más integrados al G20 se verán perjudicados. Para la OCDE, el porcentaje del PIB de EE.UU. que está en riesgo es del 1,5\% y para China es del 4,3\% (Sandri, 2019).

Para Colombia, la crisis global que recién empieza representará la llegada de productos chinos muy baratos, pues 740 productos que Colombia exporta serán objetos de gravámenes de entrada para los chinos, lo que da nuevas oportunidades al mercado colombiano y aumenta la productividad en sectores agrícolas, productos de tubería, vidrio, neumáticos, ñame, sacarosa y tejidos. No obstante, lo anterior puede verse aminado por el déficit de cuenta corriente, fiscal, pago de dividendos Ecopetrol y la consecuente subida del dólar frente al peso colombiano. Sin duda, los mercados emergentes y las monedas continuarán depreciándose de continuar el enfrentamiento bipolar, presentado al mundo como uno de tinte económico (Lioman, 2019).

\section{Conclusiones}

La corrupción es el cáncer del sistema colombiano. La falta de transparencia en sectores de administración pública, facilitó y facilita la expansión de los actores del conflicto armado hacia las esferas de actuación pública. Todo ello hace aún más complejo el escenario 
interno colombiano, que afronta un reto inmenso luego de la firma de los Acuerdos del Colón, pues existen plataformas de construcción antes no exploradas para armonizar las relaciones sociales y develar un desarrollo económico importante en el futuro.

El proceso de paz negativa iniciado en Colombia representa un escenario positivo para incrementar las inversiones en sectores de exportaciones no tradicionales como el turismo, así como se espera un mayor desarrollo en los campos agrícolas, mineros, petroleros, energéticos y los relativos a sus propias infraestructuras. El principal reto de la administración pública es la efectiva implementación o, al menos, la implementación gradual de las buenas prácticas en el escenario post-conflictual.

En el presente artículo hemos demostrado cómo, de manera tangible, el Estado colombiano durante la administración de Juan Manuel Santos Calderón se preocupó por diseñar un plan de acercamiento a la OCDE hasta lograr formalizar su invitación de ingreso el pasado mayo de 2018, y realizó acciones concretas para cuantificar y cualificar los diversos sectores posibles de desarrollo y cooperación con el Estado colombiano.

Sin estar cerca de considerarnos un país de buenas prácticas, podemos sin embargo establecer que los procedimientos públicos mejoraron parcialmente así como también, a nivel normativo, sus estándares de efectividad y transparencia. Esto también se debió a las exigencias globales. No obstante, es condición sine qua non para continuar el desarrollo del país implementar cada vez más herramientas que disminuyan los actos sistemáticos de corrupción, en especial, en el interior de las estructuras administrativas, teniendo en cuenta que somos uno de los países más corruptos del mundo.

En el sector económico, el Estado colombiano ha sabido acoplarse a las caídas de los precios mundiales de las materias primas. Igualmente ha sabido manejarse en el diálogo internacional con los gremios comerciales.

El intercambio de información más fluido ha permitido diversificar los escenarios productivos, pese a que ellos no resultan suficientes en cuanto su autosostenibilidad y rendimientos. Una alianza estratégica ha sido el acercamiento del Ejecutivo a los gremios de la producción, lo que ha logrado buscar respuestas comunes a los desafíos sectoriales. Esta alianza debe estrecharse en mayor grado hasta alcanzar la materialización de resultados recíprocos de mayor impacto a nivel de diversificación productiva que impulsen el dinamismo económico y puedan cuantificarse objetivamente a través de los ascensos que se presenten en el PIB per capita.

Al aumentar y diversificar la productividad se multiplican gradualmente las opciones de crecimiento socio-económico sostenido. Para lograrlo es necesario estabilizar los niveles del gasto público, lo que facilitará la distribución equitativa de la riqueza. Otro aspecto clave para incrementar la productividad es concentrarse en ofrecer productos de valor agregado.

Otro de los males del sistema colombiano que alimenta el cáncer de la corrupción es la altísima desigualdad entre sus ciudadanos ricos y pobres, lo que requiere urgentemente del diseño y aplicación de políticas sociales de consideración redistributiva. Esta desigualdad desmedida daña la cohesión nacional, lo que conlleva un verdadero desafío político a enfrentar. También es evidente la necesidad vital de garantizar los derechos humanos como requisito fundamental para conseguir la sostenibilidad de la paz.

Fundamentales serán las decisiones que se adopten en materia técnica y financiera, las que deberán adaptarse a las políticas económicas, pues se entiende que estas requieren de dinamismo y de análisis de riesgos contundentes. Colombia está muy expuesta a los cambios que se presentan por causa de las expectativas del mercado financiero mundial ya que 
tiene un elevado déficit de cuenta corriente, lo que hace al país dependiente de dichos flujos de cartera, sin importar las amplias deudas o créditos externos, como los que se adeudan con el Fondo Monetario Internacional y que, sin duda, erosionan la confianza mercantil.

Otra de las necesidades será además apegarse a las posibilidades que ofrecen las plataformas tecnológicas para mejorar en todos los sectores de trabajo directo que se propone la OCDE, junto con los procesos de reforzamiento del capital humano y sus mejoramientos en la gestión. Seráfundamental la integración cada vez mayor de las empresas privadas y la sociedad civil, en la consecución de las metas trazadas por el Estado ante el organismo.

Es importante crear planes de contingencia frente a los sectores exportadores tradicionales y no tradicionales, debido a las amenazas de los decrecimientos de las economías de los principales socios comerciales colombianos, como lo son Estados Unidos, la Unión Europa, América Latina y China. Cabe destacar además la generación de barreras políticas a raíz del conflicto armado del postconflicto.

Otro frente de contingencia urgente y apremiante en el Estado colombiano, así como para el organismo, son los asuntos climáticos, que deben dejar de ser vistos como la piedrita en el zapato de los asuntos económicos y deben priorizárselos como el escenario vital de la humanidad.

Las anteriores consideraciones nos muestran una lectura más amplia de las implicaciones del proceso de adhesión colombiano a la OCDE, que la tradicional lectura, aunque real, de encontrarnos en escenarios de interdependencia, en donde las asimetrías configuran modelos organizacionales internacionales, que terminan afectando el derecho interno, de forma positiva y negativa.

El triunfo del neoliberalismo como modelo económico es impajaritable. Por ello, aprovechar la mejoría de la imagen internacional del Estado Colombiano puede conducirlo por un andamiaje más evolucionado de buenas prácticas, que empiecen a redundar en el mejoramiento de la calidad de vida y efectividad de los derechos humanos de los habitantes del territorio nacional, tan afectado por las necesidades más vitales insatisfechas y la violencia sistemática que no se cansa de mutar. Todo ello en pos de salir del podium de los países más desiguales del planeta.

\section{Referencias}

ACNUR (2016). "Tendencias globales sobre refugiados y otras personas de interés del ACNUR." Recuperado el 26 de octubre de 2017 de http://www.acnur. org/recursos/estadisticas/

Aministía Internacional (2017). https://www.amnesty.org/es/countries/americas/colombia/report-colombia/

Antolinez, V. (2018). "Economía colombiana crecerá en 2019: OCDE”. https://www.lafm.com.co/economia/economia-colombiana-crecera-en-2019ocde. Publicada el 21 de noviembre y consultada el 27 de febrero de 2019.

Arias Henao, D. (2008). "Sistema Político Colombiano: el desarrollo de la violencia y la incidencia del condicionante externo". Instituto de Relaciones Internacionales de la Universidad Nacional de La Plata. http://se- 
dici.unlp.edu.ar/bitstream/handle/10915/1806/Documento completo .pdf?sequence $=1$. Publicado en mayo de 2008 y consultado el 8 de marzo de 2019.

Arias Henao, D. Y J. Idrobo Velasco. (2018). "Crimen social, justicia transicional y territorio. Apuntes críticos sobre la intervención del "Bronx" en Bogotá". Revista Reflexión Política. Año. 20 No 40. Diciembre. ISSN (en línea) 2590-8669; IEP UNAB, Bogotá, Colombia. https://revistas.unab.edu.co/index.php/reflexion/article/view/3446/2937. Doi https://doi.org/10.29375/01240781.3446.

BBC News. (2019). “Guerra comercial entre China y Estados Unidos: cómo la maquinaria de propaganda de Pekín presenta la disputa con Washington por los aranceles". Mundo, 16 mayo de 2019. https://www.bbc.com/mundo/noticias-internacional-48261034.

Bergemann, A. \& G. Van der Berg. (2008). "Active Labor Market Policy effects for Women in Europe - A Survey". Annals of Economics and Statistics, 2008, Issue 91-92, pp. 385-408.

Bell \& Schipani. (2015). "Colombia prioritises infrastructure plans. Roads, bridges and tunnels are top priorities. Investing together in the Pacific Alliance Countries." Financial Times.

Botero, R. (2019). “Colombia y la Ocde.” https://www.larepublica.co/analisis/rodrigo-boteromontoya-500048/colombia-y-la-ocde-2858294. 4 de mayo de 2019.

Brown, J.D., G. Crespi, L. lacovone \& L. Marcolin. (2016) "Productivity Convergence at the Firm Level: New Evidence from the Americas". En: Thomson Araujo, J. et al. (eds.) Understanding the Income and Efficiency Gap in Latin America and the Caribbean. Directions in development; countries and regions. Washington, D.C. : World Bank Group.

Causa, O. \& Å. Johansson. (2009). "Intergenerational Social Mobility". OECD Economics Department Working Papers, No. 707, OECD Publishing, Paris.

Central Bank of Colombia. (2016). "Reporte de Estabilidad Financiera”, septiembre.

CM\&. (2019). https://canal1.com.co/noticias/economia/la-ocde-vuelve-reducir-su-previsionde-crecimiento-mundial-3-3-para-2019/06-Mar-19. Redacción Internet. Consultado el 11 de marzo de 2019.

Cournede, B., O. Denk \& P. Garda. (2016). "Effects of Flexibility-Enhancing Reforms on Employment Transitions", OECD Economics Department Working Paper, No. 1348.

DANE. (2019). https://www.dane.gov.co/index.php/estadisticas-por-tema/mercado-laboral/empleo-y-desempleo. Consultado el 8 de marzo de 2019.

Dougherty, S. \& O. Escobar. (2013). "The Determinants of Informality in Mexico's States", OECD Economics Department Working Paper No. 1043.

El Mundo. (2019). "Google volverá a colaborar con Huawei durante el indulto de Trump al fabricante chino." https://www.elmundo.es/economia/empre- 
sas/2019/05/21/5ce4336a21efa0c5308b4602.html. 21 de mayo. Madrid, España.

Fan. (2004). "Infrastructure and Pro-poor Growth", Paper for OECD DACT POVNET workshop, Helsinki.

Foucault, M. (1998). "El sujeto y el poder". En: Revista Mexicana de Sociología, 50(3), pp. 320.

Frey, V., A. Jaoui \& T. Manfredi. (2017). “Gender inequality in Colombia vis-à-vis OECD countries". En: Economics Department Technical Background Paper.

CPAT \& Parces ONG. (2017). "Destapando la olla. Informe sombra sobre la intervención en el Bronx: Informe Sombra sobre la intervención en el Bronx." Recuperado de http://cpatong.com/ destapandolaolla/49

Heckman, J. (2006). "Skill Formation and the Economics of investing in Disadvantaged Children”, Science, Vol. 312, No. 5782, pp. 1900-02.

Hummels, D., V. Lugovskyy \& A. Skiba. (2009). “The Trade Reducing Effects of Market Power in International Shipping". En: Journal of Development Economics, Vol. 89, No. 1: 84-97.

Karpowicz, I. (2014), “Financial Inclusion, Growth and Inequality: A Model Application to Colombia". IMF Working Paper No. 166.

Lioman, L. (2019). "Guerra comercial entre China y Estados Unidos: cómo la maquinaria de propaganda de Pekín presenta la disputa con Washington por los aranceles." https://www.eltiempo.com/economia/sectores/como-afecta-a-colombia-laguerra-comercial-de-estados-unidos-y-china-360334 BBC News Mundo, 16 de mayo.

Loayza, N.V., L. Serven \& N. Sugawara. (2009). "Informality in Latin America and the Caribbean". World Bank Policy Research Working Paper Series, No. 4888.

Lozano-Espitia \& Ramirez-Villegas. (2016). “How Productive is Rural Infrastructure? Evidence on Some Agricultural Crops in Colombia”. En: Borradores de Economia Núm. 948.

Morales, C. (2019). En Colombia hay poco más de 1 millón 260 mil venezolanos. https://www.eltiempo.com/mundo/venezuela/cifra-de-venezolanos-en-colombia-a-corte-del-31-de-marzo-de-2019-356426. 3 de mayo 2019. Bogotá, Colombia.

Nuevo Siglo. (2019). https://www.elnuevosiglo.com.co/articulos/02-2019-ocde-revela-fallasde-la-economia-colombiana. Consultado el 8 de marzo de 2019.

OECD. (2009). Clusters, Innovation and Entrepreneurship, LEED Reports.

OECD. (2015). "The Future of Productivity". OECD Publishing, Paris, http://dx.doi.org/10.1787/9789264248533-en. OECD (2015c), Universal Basic Skills: What Countries Stand to Gain, OECD Publishing, Paris.

OECD. (2017). “Estudios Económicos de la OCDE Colombia”. Visión general extraída del Estu- 
dio Económico de la OCDE de Colombia 2017.http://www.oecd.org/eco/surveys/Colombia-2017-OECD-economic-survey-overview-spanish.pdf. Publicado en mayo de 2017 y consutado el 27 de febrero de 2019.

OCDE. (2018). "Colombia en la OCDE: las realidades de su adhesión." http://www.oecd.org/countries/colombia/lanzamiento-del-documento-demejores-politicas-para-colombia-en-el-marco-de-la-visita-del-presidente-decolombia-juan-manuel-santos-a-francia.htm [fecha de consulta: mayo 3 de 2018].

OCDE. (2019). http://www.oecd.org/centrodemexico/laocde/colombia-y-la-ocde.htm. Consultado el 27 de febrero de 2019.

ONU. (2019). "Informe Anual del Alto Comisionado para la Paz en Colombia." Informe anual 17 http://www.hchr.org.co/documentoseinformes/informes/altocomisionado/Informe-anual-colombia-2018-ESP.pdf. marzo.

Pinstrup-Anderson, P. \& S. Shimokawa. (2006). "Rural Infrastructure and Agricultural Development", Paper for Annual Bank Conference on Development Economics, Japan.

Portafolio. (2019). "Ya están firmados los términos de adhesión de Colombia a la OCDE". https://www.portafolio.co/economia/ya-estan-firmados-los-terminos-de-laadhesion-de-colombia-a-la-ocde-525286. [Fecha de publicación: el 16/01/2019. Fecha de consulta: 27/02/2019]

Portafolio. (2019). La participación de Colombia en la OCDE aún no despega. https://www.portafolio.co/economia/la-participacion-de-colombia-en-laocde-aun-no-despega-527168. Publicado el 6 de marzo de 2019. Consultado el 8 de marzo de 2019.

Portafolio, (2019b). La Ocde urge terminar el ingreso de Colombia antes de finalizar mayo. https://www.portafolio.co/economia/la-ocde-urge-terminar-el-ingreso-decolombia-antes-de-finalizar-mayo-527392. marzo 12 de 2019

Sanchez, Galindo \& Restrepo. (2009) “Conflicto y pobreza en Colombia: un enfoque institucionalista".

Sanchez A. and M. Diaz. (2005). "Los efectos del conflicto armado en el desarrollo social colombiano, 1990-2002", Documentos cede, No. 2005-58, Bogotá: cede, Universidad de los Andes.

Sandri, P. (2019). Una guerra comercial autodestructivaEE.UU. y China sufren un impacto económico negativo tras el aumento de los aranceles a sus intercambios. https://www.lavanguardia.com/economia/20190513/462196187851/estados-unidos-china-guerra-comercial.html. Barcelona, España. 13 de mayo.

Santa Maria, Rojas \& Hernandez (2013), “ Crecimiento económico y Conflicto Armado en Colombia”. Departamento Nacional de Planeacion, Direccion de Estudios Economicos, Bogota: DNP.

Serrano, A. (2015). "América latina y el Consenso de Beijing. Revista Correo del ALBA". https://www.celag.org/america-latina-y-el-consenso-de-beijing-por-alfredoserrano-mancilla/. 29 de julio. 
Vargas Alzate, L. (2018). “Colombia en la OCDE. Las realidades de su adhesión.” Fundación Konrad Adenauer, KAS, Colombia. Universidad EAFIT. Mayo. Colombia. https://www.kas.de/c/document library/get_file?uuid=23024472-ecee-f6aa64c5-a0077eafca3b\&groupld=252038

Villar, L. (2017). "El consenso de Beijing". https://www.eltiempo.com/opinion/columnistas/leopoldo-villar-borda/el-consenso-de-beijing-esta-tomando-fuerza-en-elmundo-158180. 04 de diciembre 2017

Zerda Sarmiento, A. (2018). "El ingreso de Colombia a la Ocde: oportunidad o riesgo". UN Peródico Digital. unperiodico bog@unal.edu.co. Publicado el 9 de enero y consultado el 27 de febrero de 2019. Bogotá, Colombia. 